\title{
Correction to: Association between expanded criteria for living kidney donors and renal biopsy findings
}

\author{
Shunsuke Goto ${ }^{1} \cdot$ Hideyo Oguchi $^{2} \cdot$ Ken Sakai $^{2} \cdot$ Tetsuo Mikami $^{3} \cdot$ Daisuke Ichikawa ${ }^{4} \cdot$ Masahiko Yazawa $^{4}$. \\ Junki Koike ${ }^{5}$ Kengo Furuichi ${ }^{6} \cdot$ Masahiko Kawabata $^{7} \cdot$ Hitoshi Yokoyama $^{6} \cdot$ Tadashi Sofue $^{8} \cdot$ Emi Ibuki $^{9}$. \\ Shinichi Nishi ${ }^{1}$
}

Published online: 17 February 2022

(c) The Author(s) under exclusive licence to Italian Society of Nephrology 2022

\section{Correction to: Journal of Nephrology}

https://doi.org/10.1007/s40620-021-01228-2

The originally published article the numbers in the supplementary Figures S2, S4, S5 are having errors and it has been updated.

The original article has been corrected.

Supplementary Information The online version contains supplementary material available at https://doi.org/10.1007/s40620-022-01285-1.

Publisher's Note Springer Nature remains neutral with regard to jurisdictional claims in published maps and institutional affiliations.

The original article can be found online at https://doi.org/10.1007/ s40620-021-01228-2.

Shunsuke Goto

sgoto@med.kobe-u.ac.jp

1 Division of Nephrology and Kidney Center, Kobe University Graduate School of Medicine, 7-5-2 Kusunoki-cho, Chuo-ku, Kobe 650-0017, Japan

2 Department of Nephrology, Faculty of Medicine, Toho University, Tokyo, Japan

3 Department of Pathology, Faculty of Medicine, Toho University, Tokyo, Japan

4 Division of Nephrology and Hypertension, Department of Internal Medicine, St. Marianna University School of Medicine, Kawasaki, Japan
5 Department of Diagnostic Pathology, St. Marianna University School of Medicine, Kawasaki, Japan

6 Department of Nephrology, Kanazawa Medical University School of Medicine, Uchinada, Japan

7 Department of Internal Medicine, Toyama Prefectural Central Hospital, Toyama, Japan

8 Division of Nephrology and Dialysis, Department of Cardiorenal and Cerebrovascular Medicine, Kagawa University, Kagawa, Japan

9 Department of Diagnostic Pathology, Kagawa University, Kagawa, Japan 